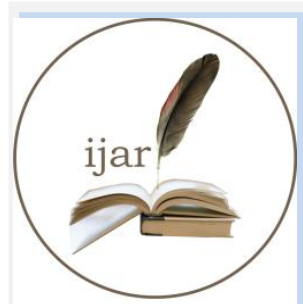

\section{Journal Homepage: - $\underline{w w w . j o u r n a l i j a r . c o m}$ \\ INTERNATIONAL JOURNAL OF ADVANCED RESEARCH (IJAR)}

DOI: $10.21474 / \mathrm{IJAR} 01 / 1229$

DOI URL: http://dx.doi.org/10.21474/IJAR01/1229

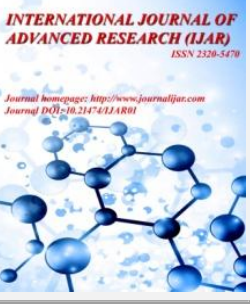

RESEARCH ARTICLE

\title{
EVALUATION OF FASCIN AND MMP-9 EXPRESSION AS MARKERS OF INVASION IN UROTHELIAL CARCINOMA OF THE URINARY BLADDER.
}

\author{
"Doaa Abdelaziz Ibrahim ${ }^{1}$, Eman H. Abdelbary ${ }^{1}$ and Abdelmonem A. Hegazy ${ }^{2}$. \\ 1. Department of Pathology, Faculty of Medicine, Zagazig University, Zagazig 44519, Egypt. \\ 2. Department of Anatomy and Embryology, Faculty of Medicine, Zagazig University, Zagazig 44519, Egypt.
}

\section{Manuscript Info}

Manuscript History

Received: 22 June 2016

Final Accepted: 26 July 2016

Published: August 2016

Key words:-

Fascin, MMP-9, Urothelial carcinoma, Immunohistochemistry.

\begin{abstract}
Background:Fascin and matrix metalloproteinase-9 (MMP-9) have been implicated in regulation of cell invasion and metastasis in many types of cancers.

Aim: To evaluate the expression of fascin and MMP-9 in urothelial carcinoma (UC) of the urinary bladder and correlate their expression with the clinicopathological variables and to assess the relationship between them.

Materials and methods: Fascin and MMP-9 immunoexpression was evaluated semiquantitatively in 60 cases of UCs according to the percentage of the positive cells.

Results:Fascin and MMP-9 expression was observed in $66.7 \%$ and $60 \%$ of the studied cases of UCs respectively. The expression was significantly different from the normal urothelium. Fascin and MMP-9 expression was significantly higher with advanced tumor stage $(P$ $=0.02$ and $=0.01$ respectively) and lymph-vascular space invasion (LVSI) $(P<0.001$ and $=0.03$ respectively). MMP-9 overexpression was significantly associated with the tumor grade $(P=0.03)$. There was a positive correlation between fascin and MMP-9 expression $(\mathrm{r}=0.5, P$ $=0.03$ ). More intense immunostaining was detected at the invasive fronts compared with other areas of the tumor in 50\% and $40 \%$ of UCs with positive fascin and MMP-9 expression respectively.

Conclusion: Our results point to an association between increased fascin and MMP-9 expression and UC invasiveness and suggest that both markers may act in concert to mediate a more aggressive behavior through promoting tumor cell invasion.
\end{abstract}

Copy Right, IJAR, 2016,. All rights reserved.

\section{Introduction:-}

Bladder cancer ranks ninth malignancy worldwide (1). In Egypt, it represents the most common cancer of the urinary system and accounted for $12.22 \%$ of total malignant tumors according to the national cancer institute (NCI) registry $(2,3)$. Bladder cancer has one of the highest rates of mortality among urogenital cancers (4). 
The biological behavior of UC depends mainly on the degree of differentiation of the tumor and the depth of invasion into the bladder wall. However, the development of reliable molecular markers may provide useful information for improving the diagnosis, delineating the treatment strategy and predicting the subsequent clinical outcome of bladder cancer (5).

The majority of patients usually present with papillary non-muscle-invasive (stage Ta, T1) UC. They may show local recurrence but infrequently progress to muscle invasion or metastasis $(4,6)$. However, patients with bladder cancers invading the muscle layer develop a high risk of local invasion and distant metastasis (4). Recent advances in molecular biology could help to identify genetic and epigenetic determinants for tumor invasion and metastasis (6).

Fascin, also known as fascin-1 is a 55-kDa actin-bundling protein that plays an important role in cell motility, migration and adhesion. Three forms of fascin proteins exist. Fascin-1 is expressed by nervous and mesenchymal tissue. Fascin-2 is expressed in the retinal photoreceptor cells while fascin-3 is expressed in testis (7). Fascin is overexpressed in a variety of tumors including esophageal (8), colorectal (9) and ovarian carcinomas(10). However, it is usually absent or downregulated in normal epithelia (7). Several investigators have reported that fascin overexpression may correlate with invasion, metastasis and poor prognosis in several types of tumors $(11,12)$.

Degradation of the extracellular matrix (ECM) is considered a critical step in tumor invasion and metastasis. A variety of proteolytic enzymes are known to degrade ECM such as plasmin, cathepsins and matrix metalloproteinases (MMPs) (13). MMP-9 is a 92-kDa zinc-dependent peptidase that belongs to the gelatinase subfamily of MMPs. MMP-9 can catalyze type IV collagen in the basement membrane, and has an essential role in tumor invasion and metastasis (14). MMP-9 overexpression was observed in tumor tissue exhibiting aggressive characteristics and low overall survival $(13,14)$. Many studies reported its correlation with tumor stage, grade and prognosis. MMP-9 overexpression was reported in several tumors including cervical (13), colorectal (14), ovarian (15) and breast cancers (16).

This study aimed to determine the expression of fascin and MMP-9 in UC of the urinary bladder and correlate their expression with clinicopathological variables and to assess the relationship between these two proteins.

\section{Materials and methods:- \\ Specimens' collection}

This study was conducted on sixty formalin fixed, paraffin embedded tissue sections of UCs of the bladder. Archival materials were obtained from Pathology Department, Zagazig University over a period of four years (2010 to 2014). Tumor specimens were obtained by transuretheral resection of bladder tumor (TURBT) (35 cases) or radical cystectomy (25 cases). Hematoxylin and eosin stained slides were examined to re-evaluate the histopathologic diagnosis. Tumors were graded according to WHO 2004 grading system (17). The TNM system was used for pathologic staging: Ta, noninvasive papillary UCs; T1, tumor invades subepithelial connective tissue; T2, tumor invades muscularispropria; and T3, tumor invades perivesical tissue (18). Fifteen normal bladder tissue samples were also included in this study. They were obtained from cystectomy specimens resected for nonneoplastic or nonurologic diseases.

\section{Immunohistochemical staining}

Sections of 3-5 $\mu \mathrm{m}$ thick, cut from formalin fixed, paraffin-embedded samples were deparaffinizedin xylene, and rehydrated through graded alcohols. For antigen retrieval, the slides were placed in sodium citrate buffer ( $0.01 \mathrm{M}$, $\mathrm{pH}$ 6.0) in a microwave for $20 \mathrm{~min}$ then washed in phosphate buffered saline ( $\mathrm{pH} 7.4)$. Endogenous peroxidase activity was blocked by incubation with $3 \%$ hydrogen peroxide for $30 \mathrm{~min}$. The sections were incubated with primary mouse monoclonal antibody against fascin (Clone: FCN01; Thermo Fisher Scientific Inc., Fremont, CA) at 1:200 dilution and mouse monoclonal anti MMP-9 (Clone: GE-213; Thermo Fisher Scientific Inc., Fremont, CA) at 1:200 dilution overnight at $4^{\circ} \mathrm{C}$. Sections were incubated with biotinylated secondary antibodies for 30 minutes. This is followed by incubation with streptavidin-biotin-peroxidase complex. The slides were rinsed with phosphate buffered saline and incubated with diaminobenzidine for 15 minutes. The slides were counterstained with hematoxylin, dehydrated, cleared and mounted.

Sections of Hodgkin's lymphoma and lung adenocarcinoma were used as positive control for fascin and MMP-9 respectively. For negative control, we substituted the primary antibody with phosphate buffered saline. 


\section{Evaluation of fascin and MMP-9 immunostaining}

Positive immunoreactivity offascin and MMP-9 was detected as brown cytoplasmic staining. Fascin and MMP-9 expression was evaluated according to the percentage of positive cells in at least five fields at a magnification of 400x. andwas scored as: 0 , negative; 1 , focal(1-10\% positive cells); 2 , moderately positive (11-50\%); and 3, strong positive $(>50 \%)$. For statistical purpose, only cases with scores 2 or 3 were considered positive cases in this study (19). Next, each marker was compared to the clinicopathological parameters.

\section{Statistical Analysis}

SPSS software version 22.0 (IBM SPSS Statistics for Windows, Version 22.0. Armonk, NY: IBM Corp, 2013) was used to analyze the data. Pearson's chi-square $(\chi 2)$ and Fisher's exact tests were used to examine the relationship between categorical variables. Spearman rho (r) correlation coefficient was performed to assess the correlation between the two markers. In all the tests, $p$ value of $<0.05$ was considered statistically significant.

\section{Results:- \\ Clinicopathologic features}

The age of the patients ranged from 46 to 70 years (mean \pm SD, $60.39 \pm 9.63)$. Among 60 cases, 50 (83.3\%) were males and $10(16.7 \%)$ females with an average male to female ratio of 5:1. Twenty five cases displayed low-grade morphology while 35 were of high grade UCs. Considering the pathologic stages, 18 cases were of stage pTa, 12 of pT1 and 30 of pT2-3. Only 4 cases were of stage pT3 in the pT2-3 group.

\section{Immunohistochemical expression of fascin}

Fascin expression was detected in $40 / 60(66.7 \%)$ of the studied cases of UCs. However, fascin was almost undetectable in normal urothelium $(\mathrm{p}<0.001)$ (Table 1, Fig. 1). The positive expression rates of fascin was also significantly higher in invasive UC stages pT1 and pT2-3 than in noninvasive UCs stage pTa $(P=0.02)$. Of the stage pTa papillary UCs, $44.4 \%$ displayed positive fascin expression in tumor cells. In contrast, $58.3 \%$ and $83.3 \%$ of stage pT1 and pT2-3 tumors respectively, demonstrated positive expression of fascin. Fascin expression was found to be significantly correlated with LVSI $(P<0.001)$. However, no statistical association was found between fascin expression and tumor grade $(P=0.5)$ as well as the other clinicopathological characteristics (Table 2). An interesting finding was that in 50\% of UCs with positive fascin expression, tumor cells at the invasive fronts expressed more intense immunostaining compared with other areas (Fig.1d).

\section{Immunohistochemical expression of MMP-9}

The present study demonstrated that MMP-9 was overexpressed in UCs compared with normal urothelium $(\mathrm{P}<0.001)$. MMP-9 was detected in 36/60 (60\%) of UCs (Table 1, Fig. 2). Statistical analysis demonstrated a significant positive association between MMP-9 positive expression rates and LVSI and pathological stage ( $P=0.03$ and $=0.01$ respectively). Non-invasive UC (pTa) showed MMP-9 expression in (33.3\%) compared to (50\%) in PT1 and $(80 \%)$ in muscle invasive tumors (pT2-3). High-grade tumors exhibited significantly higher MMP-9 expression compared to low grade tumors $(P=0.03)$. No significant associations was detected between MMP-9 expression and other clinicopathological characteristics (Table 2). Forty percent of UCs with positive MMP-9 expression showed more intense immunostaining at the invasive fronts compared with other areas.

\section{Correlation between fascin and MMP-9 expression in $60 \mathrm{UCs}$}

Based on a correlation analysis between the results of expression of fascin and MMP-9 among the studied 60 UCs, a significant positive correlation was found between the expression of fascin and MMP-9 proteins (Spearman correlation) (r) $=0.5, P=0.03$ (Table 3 ). 
Table 1Fascin and MMP-9 expression in UC and control group.

\begin{tabular}{|l|l|l|l|}
\hline & Control $(\mathrm{n}=15)$ & $\mathrm{UC}(\mathrm{n}=60)$ & $P$ value \\
\hline Fascin & & & \\
Negative & $13(86.7 \%)$ & $20(33.3 \%)$ & $<0.001 *$ \\
Positive & $2(13.3 \%)$ & $40(66.7 \%)$ & \\
\hline MMP-9 & & $24(40.0 \%)$ & $<0.001 *$ \\
Negative & $14(93.3 \%)$ & $36(60.0 \%)$ & \\
Positive & $1(6.7 \%)$ & \\
\hline
\end{tabular}

$* P<0.05$; statistically significant.

Table 2 Correlation between fascin and MMP-9 expression and clinicopathological characteristics in 60 UCs.

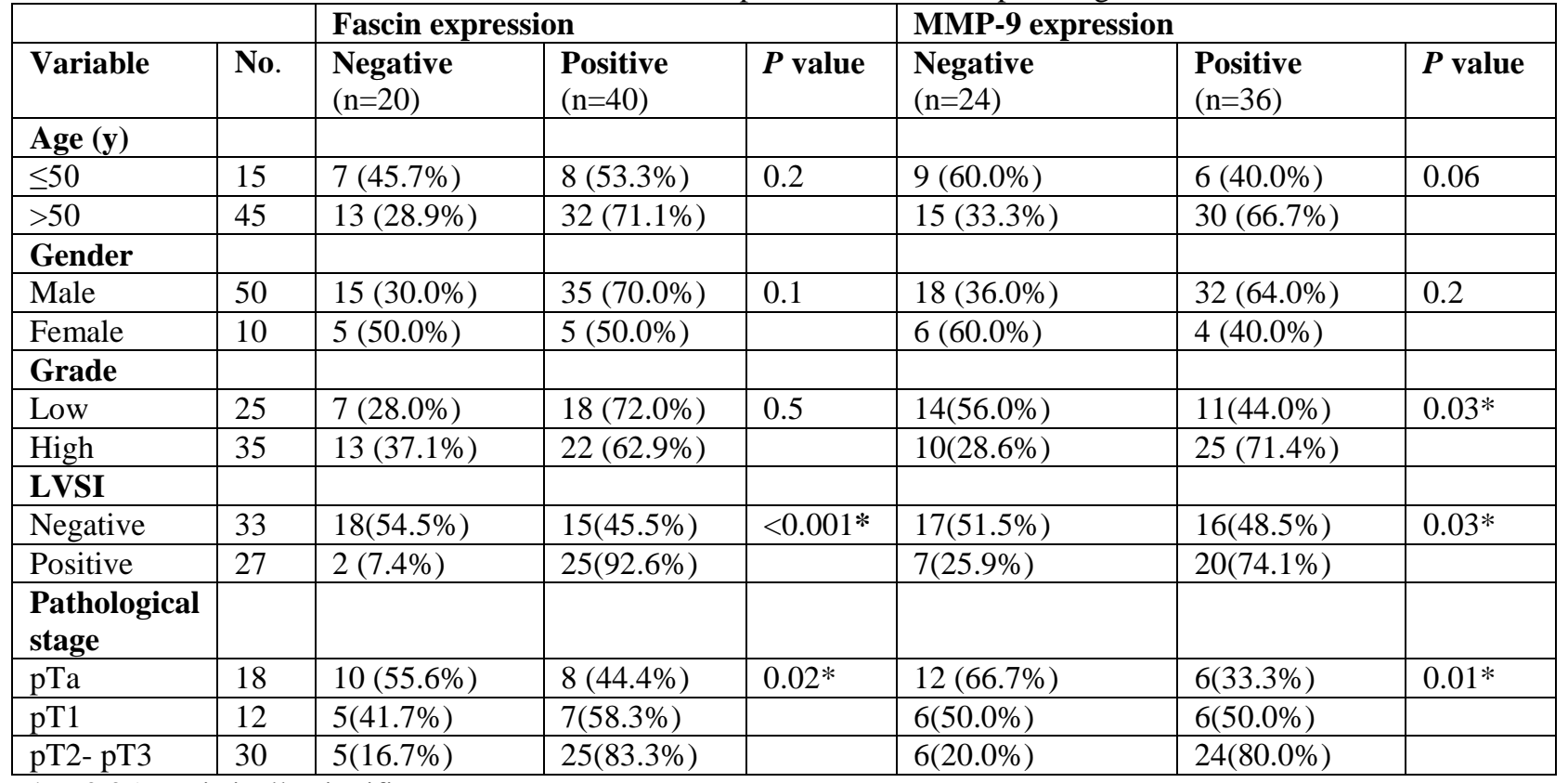

$* P<0.05$; statistically significant.

Table 3 Correlation analysis between fascin and MMP-9 expression among the studied 60 UCs

\begin{tabular}{|l|l|l|l|}
\hline & \multicolumn{3}{|c|}{ MMP-9 expression } \\
\hline & & Negative $(\mathrm{n}=24)$ & Positive(n $=36)$ \\
\hline \multirow{2}{*}{ Fascin expression } & Negative $(\mathrm{n}=20)$ & $16(80.0 \%)$ & $4(20.0 \%)$ \\
\cline { 2 - 4 } & Positive $(\mathrm{n}=40)$ & $8(20.0 \%)$ & $32(80.0 \%)$ \\
\hline
\end{tabular}

Spearman correlation $(\mathrm{r})=0.5 \quad P$ value $=0.03^{*}$

$* P<0.05$; statistically significant. 

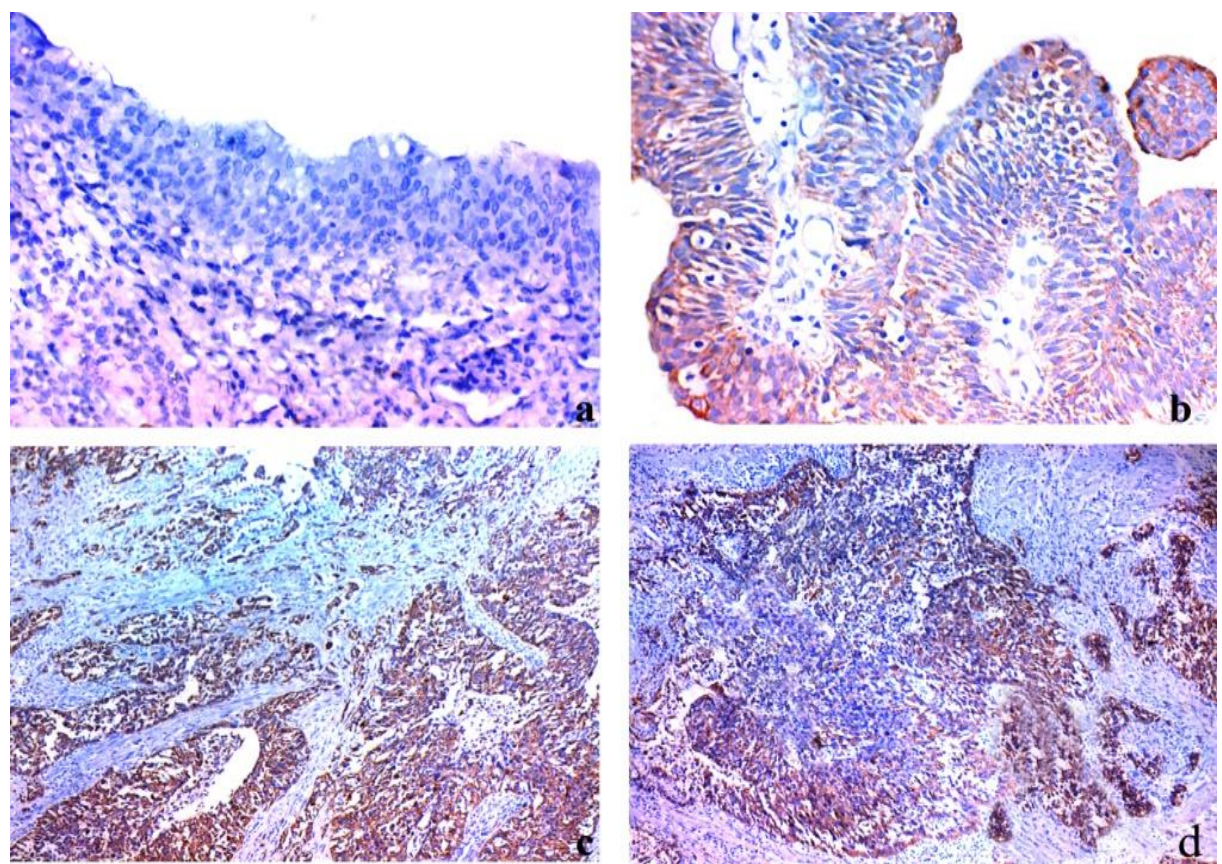

Figure 1:Fascinimmunostaining: (a) Normal urothelium negative for fascin $(\times 400)$; (b) Noninvasive (pTa) papillary UC demonstrating weak fascinimmunostaining $(\times 400)$; (c) Diffuse and strong fascinimmunostaining in muscle invasive (pT2) UC ( $\times 100)$; (d) Enhanced fascin expression at the invasive margins of the tumor $(\times 200)$.
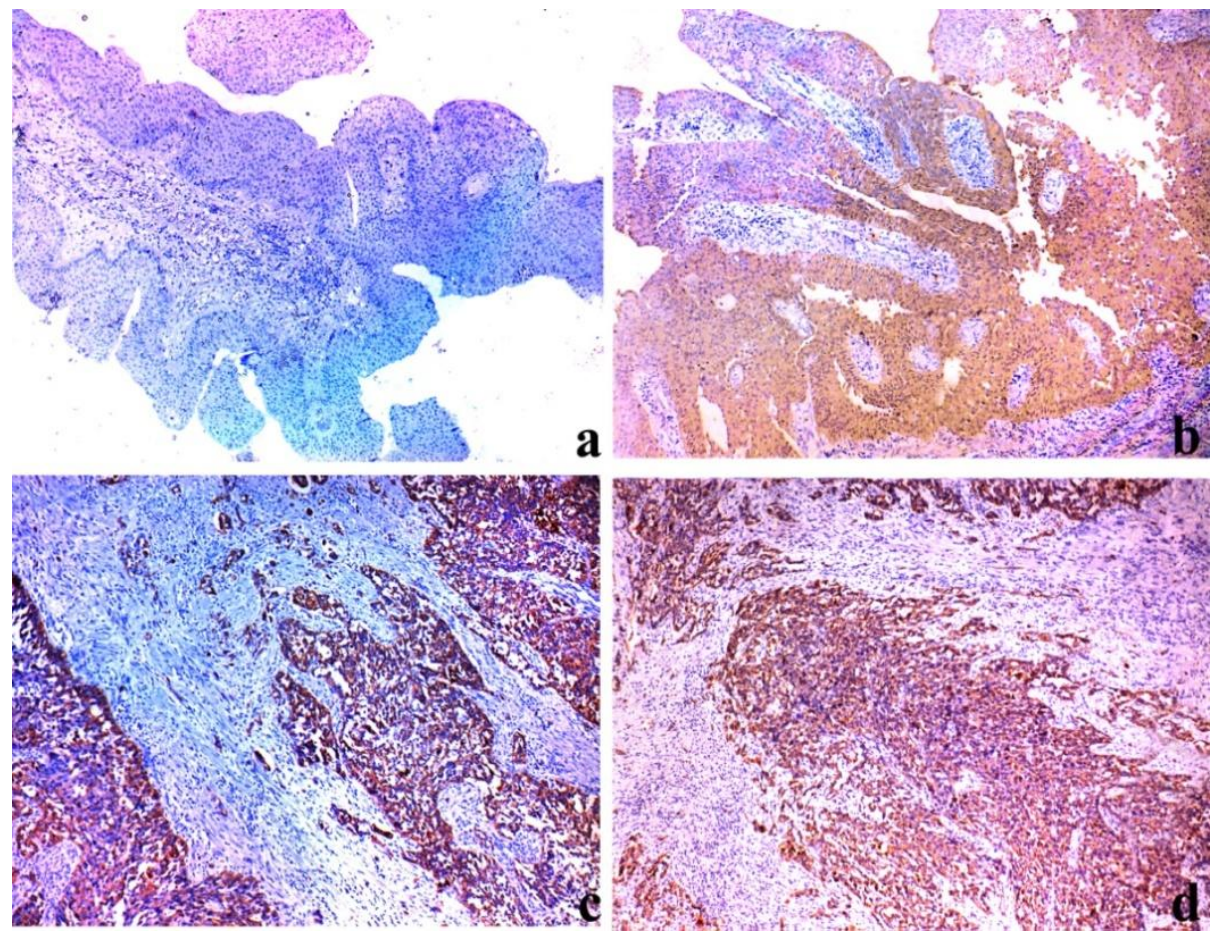

Figure 2: MMP-9 immunostaining: (a) Noninvasive (pTa) papillary UC demonstrating negative MMP-9 immunostaining ( $\times 400)$; (b) Moderate staining in invasive (pT1) UC ( $\times 100)$; (c\&d) Diffuse and strong MMP-9 immunostaining in muscle invasive (pT2) UC $(\times 100)$. 


\section{Discussion:-}

Tumor invasion and metastasis remain the leading causes of cancer related mortalities (20). Currently, there is a great interest in identifying cancer biomarkers and signaling pathways that regulate this process. Throughout various stages of tumor progression, enhanced cell motility is critical for tumor invasion and subsequent dissemination or metastasis (21). Fascin is an actin-bundling motility-associated protein that plays an important role in cell-matrix adhesion, cell interactions and cell migration (22). In the present study, we examined fascin along with MMP-9 immunoexpression in $60 \mathrm{UCs}$ of the bladder.

In the current study, fascinimmunoreactivity was detected in $66.7 \%$ of UC, while it was almost undetectable in normal urothelium. These results are consistent with previous studies showing fascin overexpression in UCs $(11,23,24)$.

The present study showed that fascin expression was also significantly higher in invasive UC stages pT1 and pT2-3 than in noninvasive UC stage pTa. We also found a significant relationship between fascin expression and LVSI.

Tong et al (23) studied the expression of fascin in urothelial neoplasms for the first time in 2005. In accordance to our results, they found absentfascin expression in normal urothelium. In addition, they observed that $42 \%$ of superficial papillary UCs (Ta) displayed a weak fascin expression while 95\% of invasive UCs (T2 or higher) demonstrated diffuse strong staining for fascin. Their study indicated that fascinimmunoexpression correlates positively with the aggressiveness of the tumor (23).

In agreement, Bi et al (11) reported a significant difference in the expression of fascin between normal urothelium and bladder UC. They demonstrated increased expression of fascin in the invasive component of the tumor which progressively increased from superficial to deeply invasive tumors; and that fascin expression was positively correlated with pT stage and tumor size (11). Also, Foteini et al (7) found that fascin expression was significantly higher in invasive UCs. In addition, they observed a strong staining exclusively in invasive carcinomas and that none of the pTa tumors demonstrated intense staining (7).

In the present study, no significant association was observed between fascin expression and tumor grade or other clinicopathological parameters. A similar absence of correlation has also been reported in previous studies (11,23).

This absence of significant association between fascin expression and tumor grade noted in the current study is in agreement with the findings of previous studies $(11,23,24)$. These findings are in accordance with the hypothesis that fascin overexpression is suggested to disrupt epithelial junctions and to enhance the invasive and metastatic potential of malignant cells.

In this study, we found a significant correlation between fascin overexpression and LVSI. Additional studies have shown that fascin overexpression in tumor cells may be associated with mortality, disease progression and metastasis $(20,25,26)$.

MMP-9 is a protein involved in different steps in the extracellular matrix invasion. Many previous studies have reported MMP-9 expression in many tumors, including UCs, but the results concerning the prognostic value of MMP-9 is a still a matter of debate.

The present results revealed that MMP-9 expression was detected in $60 \%$ of UCs with weak expression in stromal cells. However, the normalurothelium did not express MMP-9. Moreover, we have shown not only that MMP-9 is overexpressed in UCs, but also that MMP-9 positive expression rates are significantly higher in cases with high grade and infiltrative tumors, two very important prognostic factors in UCs. This was in accordance with Reis et al (27), who reported the overexpression of MMP-9 with poor prognosis in cancer bladder. Donmez et al (28) and Papathoma et al (29) reported that MMP-9 expression was significantly higher in high-grade tumors than in lowgrade tumors and in invasive tumors compared to non-invasive tumors.

In a meta-analysis, Zeng et al (30) demonstrated that MMP-9 is associated with the clinicopathological features of bladder cancer, suggesting that MMP-9 may be used in combination with other tumor specific markers, which may improve the sensitivity of diagnosis and treatment of bladder cancer. 
In contrast, Vasala et al (31) could not find any association between the overexpression of MMP-9 and the tumor stage or grade and suggested that MMP-9 overexpression in UCs of the bladder could be a marker of favorable prognosis and is associated with better overall survival. Also, Durkan et al (32) and Seiler et al(33) found no significant correlation in the proportion of muscle-invasive tumors stained positively for MMP-9 compared with pTa/T1 tumors. They also reported that MMP-9 staining was also unrelated to tumor grade $(32,33)$.

Meanwhile, no correlation was found between MMP-9 expression and the other clinicopathological features. This was also supported by the results of other studies $(29,32)$.

An interesting finding in the current study is the more intense expression offascin and MMP-9 observed at the invasive margins in some areas of tumor. This may indicate that increased cell motility and decreased cell to cell adhesion play a role in stromal invasion. This finding was also reported by other studies $(7,24)$.

Another important finding of our study was the relationship between fascin and MMP-9. In their study, Xie et al (12) suggested that the potential role of fascin in promoting tumor invasiveness is mediated through increasing the cell motility as well as activation of matrix proteases MMP-2 and MMP-9. Moreover, similar results were obtained by Onodera et al (19). They also reported that the overexpression of fascin in cholangiocarcinoma might participate in their invasive growth in cooperation with MMP-9 (19). In agreement with these findings, our study revealed a significant correlation between fascin and MMP-9 expression in UCs.

This study revealed that fascin and MMP-9 expression levels increased together with tumor invasiveness and demonstrated a significant correlation between the two proteins that may act in synergistic fashion during tumor progression and invasion.Further larger studies are still needed to find out the relation of fascin and MMP-9 expression to UC patients' survival.

\section{Conclusion:-}

Fascin and MMP-9 expression were up-regulated in bladder UCs. Over-expression of fascin and MMP-9 might play an important role in enhancing tumor invasion and progression and also may have combined utility in predicting a more aggressive behavior in UCs. The correlation between MMP-9 with fascin should be evaluated on a wider scale of tumors which can provide new approaches for bladder cancer diagnosis and help the development of new targeted drugs.

\section{Acknowledgment:-}

No external funding was provided.

\section{Conflict of interest}

None.

\section{References:-}

1. Ploeg M, Aben KK, Kiemeney LA. The present and future burden of urinary bladder cancer in the world. World J Urol. 2009;27:289-293.

2. Salem HK and Mahfouz S. Changing patterns (age, incidence, and pathologic types) of schistosoma-associated bladder cancer in Egypt in the past decade. Urology 2012; 79: 379-383.

3. EL-bolkainy M, Akram M, Gouda I, et al. Pathology of Cancer, 4th ed, Tumors of urinary system, the National Cancer Institute (NCI). Cairo University. 2013, p. 250-261.

4. Netto G J. Molecular biomarkers in urothelial carcinoma of the bladder: are we there yet? Nat Rev Urol 2012; 9:41-51.

5. Cheng L, Montironi R, Davidson DD et al. Staging and reporting of urothelial carcinoma of the urinary bladder. Mod Pathol 2009; 22:70-95.

6. Kessenbrock K, Plaks V, Werb Z. Matrix metalloproteinases: regulators of the tumor microenvironment. Cell 2010;141:52-67.

7. Foteini K, Sotirios B, Dimitra P, et al. Fascin determination in urothelial carcinomas of the urinary bladder: a marker of invasiveness. Arch Pathol Lab Med 2008;132:1912-1915.

8. Hashimoto $\mathrm{Y}$, Ito $\mathrm{T}$, Inoue $\mathrm{H}$, et al. Prognostic significance of fascin over-expression in human esophageal squamous cell carcinoma. Clin Cancer Res 2005;11:2597-2605. 
9. Jawhari AU, Buda A, Jenkins M, et al. Fascin, an actin bundling protein, modulates colonic epithelial cell invasiveness and differentiation in vitro. Am J Pathol2003;162:69-80.

10. Cao D, Ji H, Ronnett BM. Expression of mesothelin, fascin, and prostate stem cell antigen in primary ovarian mucinous tumors and their utility in differentiating primary ovarian mucinous tumors from metastatic pancreatic mucinous carcinomas in the ovary. Int J GynecolPathol 2005; 24:67- 79.

11. Bi J, Chen X, Zhang Y, et al. Fascin is a predictor for invasiveness and recurrence of urothelial carcinoma of bladder. UrolOncol 2012;30: 688-694.

12. Xie JJ, Xu LY, Zhang HH, et al. Role of fascin in the proliferation and invasiveness of esophageal carcinoma cells. BiochemBiophys Res Commun 2005; 337:355-362.

13. Yu W, Liu J, Xiong X, et al. Expression of MMP-9 and CD147 in invasive squamous cell carcinoma of the uterine cervix and their implication. Pathol Res Pract 2009; 205:709-715.

14. Zeng ZS, Huang Y, Cohen AM, et al. Prediction of colorectal cancer relapse and survival via tissue RNA levels of matrix metalloproteinase-9. J ClinOncol 1996;14: 3133-3140.

15. Sillanpaa S, Anttila M, Voutilainen K, et al. Prognostic significance of matrix metalloproteinase-9 (MMP-9) in epithelial ovarian cancer. GynecolOncol 2007; 104:296-303.

16. McGowan PM, Duffy MJ. Matrix metalloproteinase expression and outcome in patients with breast cancer: analysis of a published database. Ann Oncol 2008;19:1566-1572.

17. John NE, Sauter G, Jonathan IE, Isabell AS, eds. World Health Organization Classification of Tumours. Pathology and Genetics of Tumours of the Urinary System and Male Genital Organs. Lyon: IARC Press; 2004.

18. Greene FL, Page DL, Fleming ID, et al., eds. AJCC Cancer Staging Manual. 6th ed. New York: Springer; 2002.

19. Onodera M, Zen Y, Harada K, et al. Fascin is involved in tumor necrosis factor-a-dependent production of MMP-9 in cholangiocarcinoma. Lab Invest 2009;89:1261-1274.

20. Tan VY, Lewis SJ, Adams JC, et al. Association of fascin-1 with mortality, disease progression and metastasis in carcinomas: a systematic review and meta-analysis. BMC Medicine 2013;11:52-65.

21. Insall RH, Machesky LM. Actin dynamics at the leading edge: from simple machinery to complex networks. Dev Cell 2009;17:310-322.

22. Hashimoto Y, Skacel M, Adams JC. Roles of fascin in human carcinoma motility and signaling: prospects for a novel biomarker? Int J Biochem Cell Biol2005; 37:1787-1804.

23. Tong GX, Yee H, Chiriboga L, et al. Fascin-1 expression in papillary and invasive urothelial carcinomas of the urinary bladder. Hum Pathol 2005;36: 741-746.

24. McKnight R, Cohen C, Siddiqui MT. Fascin stain as a potential marker of invasiveness in carcinomas of the urinary bladder: a retrospective study with biopsy and cytology correlation. DiagnCytopathol 2011;39:635-640.

25. Karasavvidou F, Barbanis S, Pappa D, et al. Fascin determination in urothelial carcinomas of the urinary bladder: a marker of invasiveness. Arch Pathol Lab Med 2008;132:1912-1915.

26. Sharma MA, Badwal CS,Dutta BV, et al. Evaluation of fascin-1 expression as a marker of invasion in urothelial carcinomas. Med J Armed Forces India. 2014;70:139- 143.

27. Reis ST, Leite KM, Piovesan LF, et al. Increased expression of MMP-9 and IL-8 are correlated with poor prognosis of Bladder Cancer. BMC Urology 2012;36: 12-18.

28. Donmez G, Sullu Y, Baris S, et al. Vascular endothelial growth factor (VEGF), matrix metalloproteinase-9 (MMP-9), and thrombospondin-1 (TSP-1) expression in urothelial carcinomas. Pathol Res Pract. 2009;205(12): 854-857.

29. Papathoma AS, Petraki C, Grigorakis A, et al. Prognostic significance of matrix metalloproteinases 2 and 9 in bladder cancer. Anticancer Res 2000; 20:2009-2013

30. Zeng F, Cen S, Tang Z, et al. Elevated matrix metalloproteinase-9 expression may contribute to the pathogenesis of bladder cancer. Oncology letters 2016; 11: 2213-2222.

31. Vasala K, Paakko P, Turpeenniemi-Hujanen T. Matrix Metalloproteinase-9 (MMP-9) immunoreactive proteinin urinary bladder cancer: A Marker of Favorable Prognosis.Anticancer research 2008; 28: 1757-1762.

32. Durkan GC, Nutt JE, Rajjayabun PH, et al. Alteration in urinary metalloproteinase-9 to tissue inhibitor of metalloproteinase-1 ratio predicts recurrence in non muscle-invasive bladder cancer. Clin Cancer Res 2003; 2576-2582.

33. Seiler R, Thalmann G N, Fleischmann A. MMP-2 and MMP-9 in lymph-node-positive bladder cancer. J ClinPathol 2011; 64 (12):1078-1082. 